\title{
A Highly Scalable Zone based Multicast Protocol for Redundant Overhead Minimization
}

\author{
R.Shankar \\ Research Scholar and Associate Professor, \\ Department of Computer Science and \\ Engineering, \\ Indira Institute of Engineering and Technology, \\ Tiruvallur - 631 203, Tamilnadu, India
}

\author{
E.llavarasan, Ph.D \\ Professor, \\ Research Supervisor, \\ Department of Computer Science and \\ Engineering, \\ Pondicherry Engineering College, \\ Pudhucherry-605 014, Tamilnadu, India
}

\begin{abstract}
This paper proposes a robust and energy efficient Highly Scalable Zone based Multicast Protocol (HSZMP) to achieve high scalability with reduced control overhead even under high dynamic mobility and large network size. This work constructs a virtual-zone-based structure in order to reduce the overhead for route discovery and maintenance of the tree structure. The position information of the node is utilized to construct the zone, multicast tree, and multicast packet forwarding. This work proposes a bi-directional multicast packet forwarding to forward the data packet along the constructed multicast tree with reduced transmission overhead. The proposed work dramatically improves the scalability even under large network and group size. This work introduces an adaptive zone handling technique to handle the empty zone problem under frequent topology changes. Furthermore, it deploys two novel mechanisms such as Broadcast from Beacon Only (BBO), and Redundancy Reducing Flooding Mechanism (RRFM) to reduce the redundant overhead perfectly for achieving scalability. The simulation results demonstrate that proposed HSZMP is an effective protocol compared to Scalable Position-Based Multicast (SPBM) in terms of packet delivery ratio, control overhead, and scalability.
\end{abstract}

\section{Keywords}

Mobile Ad Hoc Networks, Multicast Routing Protocol, Scalable Multicast, Group Communication

\section{INTRODUCTION}

Mobile ad hoc network (MANET) is constructed without any predetermined infrastructure. It consists of a set of mobile nodes that cooperates each other to perform communication without relaying on any assistance from base stations. In this network, a mobile node can act as a source (transmitter), destination (receiver) or an intermediate router to route the packets between the source and destination. The main notable characteristics of MANET are limited resources in terms of bandwidth, battery power and dynamic mobility. Multicasting is defined as the process of transmitting a same copy of a single data packet over a multiple number of destinations [1]. Conventional multicast protocols in MANET are classified into two types such as a tree based and mesh based protocols. Tree based multicast routing protocols includes Multicast Ad hoc On-demand Distance Vector (MAODV) [2], Ad hoc Multicast Routing protocol utilizing increasing id-numbers (AMRIS) [3], and Multicast Zone Routing Protocol (MZRP) [4] provide effective transmission of data packets, but it is hard to maintain the tree structure under a high dynamic mobility. Therefore, tree based multicast structure incurs high overhead to maintain its structure. Mesh based multicast routing protocol includes Forwarding Group Multicast Protocol (FGMP) [5], Core-Assisted Mesh Protocol (CAMP) [6], and On-Demand Multicast Routing Protocol (ODMRP) [7] enhances the efficiency and robustness of multicast transmission using the redundant path between the source and destination.

This work proposes Redundancy Reducing Flooding Mechanism in an efficient geographic multicast protocol to reduce the redundant overhead for multicast tree construction and membership management. The proposed approach constructs virtual zone based structure using the location information. It reduces the substantial overhead incurred for creating and maintaining the geographic zones over the wide network. It leads to support a more efficient, reliable and scalable multicast communication under dynamic mobility in MANET environment. The proposed multicast protocol can efficiently construct the packet distribution path. It reliably maintains the membership even under high network mobility due to the unreliable wireless channel and node movements. This paper proposes an efficient and scalable zone supported a geographic routing protocol with fully reduced maintenance overhead and routing failure. In zone construction, the proposed approach constructs a flexible zone based structure that allows nodes can enter or leave the group adaptively. In multicast forwarding, the proposed protocol forward data packets loosely along the efficient transmission path rather than overlay based multiple unicast transmissions. It achieves high scalability because multicast transmission carried out along the efficient transmission path. This approach provides a zone partitioning mechanism to partition the zone according to the network density in order to manage the empty zone problem.

\subsection{Problem Assertion}

Zone based Multicast routing protocol (ZMRP) has issues related to performance and scalability. In zone membership management, an efficient and robust mechanism is still in need to construct the zone based structure under high dynamic mobility and large network size. Further, a more effective zone representative election strategy is not yet available to elect the most cost efficient representative for zone membership management. In addition to, existing multicast protocol does not handle the empty zone problem. In multicast packet forwarding, the issues related to the efficient 
construction of a multicast path for control and multicast data transmissions are not resolved effectively. The efficient tree structure maintenance during dynamic mobility and obtaining location information without resorting to an external location server are not yet addressed. Redundant transmission of control message in the network increases overhead in the network. Flooding of control messages and periodic beaconing is the principal process to be taken into account in reducing the overhead. Both consume network resources such as energy and bandwidth. Moreover, they cause the packet collision in the network which needs retransmission with the cost of additional delay. Static beacon frequency is not applicable to the dynamic network environment. Position of high mobility node varies drastically which needs frequent location updating. On the contrary, for the low mobility nodes, it is sufficient to update the location less frequently. Customizing the beacon frequency is required to improve performance in the dynamic network environment.

\subsection{Contribution of the Paper}

The main contributions of this paper

- Multicast via Greedy Forwarding: This work proposes a bi-directional multicast packet forwarding to forward the data packet along the constructed tree in order to reach the destination. It totally reduces the communication overhead and access latency

- Group Membership management: The proposed work deploys a location-aware Quick group joining and leaving technique for more reliable membership management and packet transmissions. It dramatically improves the scalability even under large network and group size.

- Adaptive Zone Handling: This technique addresses the vital problem in a zone based protocol called as a empty zone problem by adaptively adjusting the multicast tree structure even under dynamic mobility.

- Redundancy Reducing Flooding Mechanism (RR-FM): RR-FM scheme contributes the efficient flooding scheme in flooding the control messages involved in the protocol. It reduces the redundancy using receiver list oriented message transmission to reduce the redundant overhead in the network

- Broadcast from Beacon Only (BBO) scheme: BBO scheme reduces overhead in the network by restricting the beacon broadcast only to the nodes located in the neighborhood area of the multicast tree. The beacon update frequency is customized based on the mobility characteristics of the node.

\subsection{Paper Organization}

The rest of this paper is organized as follows. Section 2 discusses the related work of existing routing protocol. Section 3 present a detailed design of the proposed highly scalable zone based multicast protocol. Finally, Section 4 offers the simulation results and concludes the paper in Section 5.

\section{RELATED WORK}

Already existing conventional topology based routing protocols incurs high overhead to construct and maintain a multicast tree or mesh structure under dynamic mobility. It is extremely difficult to scale multicast protocol over a large network size. Research efforts have been taken to design a more scalable routing protocol with less overhead even under a high mobility and long path transmission. However, it is a still hot research topic in the area of multicast routing in the network.

Location based multicast protocols are proposed in [8] [9] where multicast forwarding takes place based on the global knowledge of location information. In [8], Martin et al proposes position based multicast which is an extension of existing position-based unicast routing protocols such as face2 or Greedy Perimeter Stateless Routing Protocol (GPSR). This approach devises the rules to split the multicast packets and introduces a new repair strategy when no direct neighbor is available for forwarding the data packets to one or more destinations. It achieves a high scalability and flexibility even under a high dynamic mobility. This protocol performs well in certain environments such as inter-vehicle-communication, where nodes can easily access its geographical position using peripheral location detection devices. It reduces the high communication overhead, and achieves high scalability. Pariza et al proposes Scalable Energy Efficient Location Aware Multicast Protocol (SEELAMP) protocol [9] performs an efficient multicast routing. This approach mainly proposes a local connectivity management that improves the performance of multicast forwarding.

A lot of work has been carried out on Geocasting Multicast routing Protocol (GMP) [10], [11] other than general multicasting protocol. In geocasting, source node forwards a single packet to the set of nodes within a specified geographical region. Once reaching the packet destined region, packet will be sent to the set of destinations through flooding or any other schemes. Therefore, a group of peers with in a region forms a Geocast group dynamically according to the time. Here, the multicast tree is constructed through a centralized calculation and applicable for a smaller group with fewer nodes. This routing protocol proposes two types of location based multicast algorithms that limit the forwarding space for a multicast packet within a forwarding zone. In [11], Young et al proposes a novel Geocasting protocol by combining the Temporally Ordered Routing Algorithm (TORA) unicast routing protocol and flooding mechanism that perfectly reduces the geocast message overhead.

Finally, analysis is carried out on hierarchical group management approaches such as Hierarchical Rendezvous Point Multicast (HRPM) [12] and Scalable Position-Based Multicast (SPBM) [13] to manage the large number of nodes in the multicast tree. The key design of HRPM protocol [12] is that large groups with a number of peers are decomposed into a hierarchy of manageable-sized subgroups recursively, and hierarchy is constructed and maintained using distributed geographic hashing. In SPBM [13], the network terrain is partitioned into L-levels of a quad tree. In this terrain, a higher level consists of large squares and covering four smaller squares at the next level. Therefore, nodes in the bottom level of basic square must be within each other's transmission range. Then each square in the bottom level updates its membership information over the top level square periodically. This periodic flooding is carried out repeatedly for every two neighbor levels to maintain the whole network effectively. However, this membership maintenance significantly improves the control overhead under a large network size. A wide range of multicast routing protocols are proposed in literature, but none of the approaches does not address the mechanism to control the redundant overhead incurred through flooding under a high dynamic mobility and a large network size. 


\section{HIGHLY SCALABLE ZONE BASED MULTICAST ROUTING PROTOCOL TO MINIMIZE REDUNDANT OVERHEAD}

This paper proposes a Redundancy Reducing Flooding Mechanism in the zone based multicast routing protocol to achieve efficient and scalable multicast protocol with reduced overhead. The main objective is to reduce the incurred overhead for zone construction, membership management and multicast data forwarding. In zone construction, this approach constructs a two-tier virtual zone based structure by keeping a pre-determined virtual origin where nodes in the network are self-organized into a set of zones within a certain geographical region. In the two-tier network, zone representative act as a leader to manage the upper tier network where nodes can enter or leave dynamically into a multicast group as required. Lower tier consists of a set of nodes connects with the zone representative in its own zone. For multicast forwarding, this approach constructs a zone-based multicast tree to transmit data packets along the multicast tree. It proposes Adaptive Zone Handling to handle the empty zone problem due to dynamic mobility. The main key point of this paper is to achieve high scalability by effectively reducing the redundant overhead incurred due to flooding of control and data packets over the network. Therefore, this paper proposes a Redundancy Reducing Flooding Mechanism (RRFM) which contributes an efficient flooding scheme to flood the control messages involved in the protocol. Here, receiver list oriented message transmission is performed to reduce the redundant overhead in the network. Furthermore, it proposes a Broadcast from Beacon Only (BBO) scheme to restrict the beacon broadcast only to the nodes located in the neighborhood area of the multicast tree to reduce the overhead in the network.

\subsection{Network Setup}

The proposed approach constructs virtual zone based structure using the set of nodes $\left\{\mathrm{N}_{1}, \mathrm{~N}_{2} \ldots \ldots \ldots \mathrm{N}_{\mathrm{k}}\right\}$ based on the reference point. Further, zone representative (Zrep) is selected for each zone. In the proposed protocol, Zrep encompasses a multicast table to maintain the information of members in the zone. Suppose a source node $\mathrm{S}$ forwards a multicast packet to the Target nodes $\left(T_{1}, T_{2} \ldots \ldots \ldots T_{n}\right)$ follows a geographic forwarding strategy. It effectively decides the next hop node towards each destination. In order to forward the packet, a multicast tree (MT $=\{\mathrm{G}, \mathrm{S}, \mathrm{M}\})$ is constructed where $\mathrm{G}$ is a multicast group, $\mathrm{S}$ is a source, and $\mathrm{M}$ is a member of multicast group $\mathrm{G}$. In geographic forwarding, source node, $\mathrm{S}$ inserts the list of the next hop nodes along the multicast tree to reach the destination in its packet header. For example, the next hop list will be in the form of $\left(\mathrm{N}_{3}: \mathrm{T}_{1}, \mathrm{~T}_{4} ; \mathrm{N}_{2}: \mathrm{T}_{3}\right)$, where $\mathrm{N}_{3}$ is the next hop node for the targets $\mathrm{T}_{1}$ and $\mathrm{T}_{4}$, and $\mathrm{N}_{2}$ is the next hop node for $\mathrm{T}_{3}$. Thus, the source node can efficiently forward the data packets using location information without any complicated flooding. While receiving the packet, a neighbor node will check whether it is a next hop node or destination as mentioned in the packet header. If it is destination, then keep the packet, otherwise drop the packet. In some cases, the next hop nodes or destination is associated with downstream destination then forward the data packets to reach the destination.

\subsubsection{Zone Representative Selection}

For every zone, the zone representative is elected using the cooperation between the nodes in the zone in order to maintain the membership management consistently. Each node announces its existence by flooding the beacon message B (node id, zone ID, Flag) throughout the network for every interval $\left(\mathrm{Int}_{\max }\right)$. A zone representative is selected for each zone using the information in the announced beacon message for every interval $\left(\mathrm{Int}_{\mathrm{min}}\right)$. Therefore, each node updates its neighbor table for that interval $\left(\mathrm{Int}_{\mathrm{min}}\right)$. The zone representative is selected using any one of the following four ways as follows

1. If the zone consists of the only one node, then it is selected as a zone representative by itself.

2. In a zone, a flag bit of all of the nodes are disabled, no nodes in the zone are selected as a representative. Therefore, node is closer to the center of the zone is elected as a representative and flood a beacon message for announcing a representative role to all of the nodes in its zone.

3. In a same zone, a flag bit of all of nodes are enabling the node with the highest node ID is elected as a zone representative.

4. A node having higher alpha value is elected as a zone representative for its located zone. Thus, the zone representative has high capability to perform multicast packet forwarding among the multicast tree.

\subsection{Multicast via Greedy Forwarding}

In multicast forwarding over the constructed zone based structure, data packets will be forward within a same (intra) zone or out of the zone (inter) to reach the destination. In the constructed virtual zone-structure, the zone representative is aware of the location of all nodes in its zone and then each node in the zone also aware of the location of other nodes in transmission range. In the case of intra communication when source and destination are located within the same zone, only one transmission is needed to reach the destination. On the contrary, for inter communication when destination will be in the different zone of that source node, then the location of the destination must be tracked to ensure reliable transmission between the source and destination which is in multiple hops away.

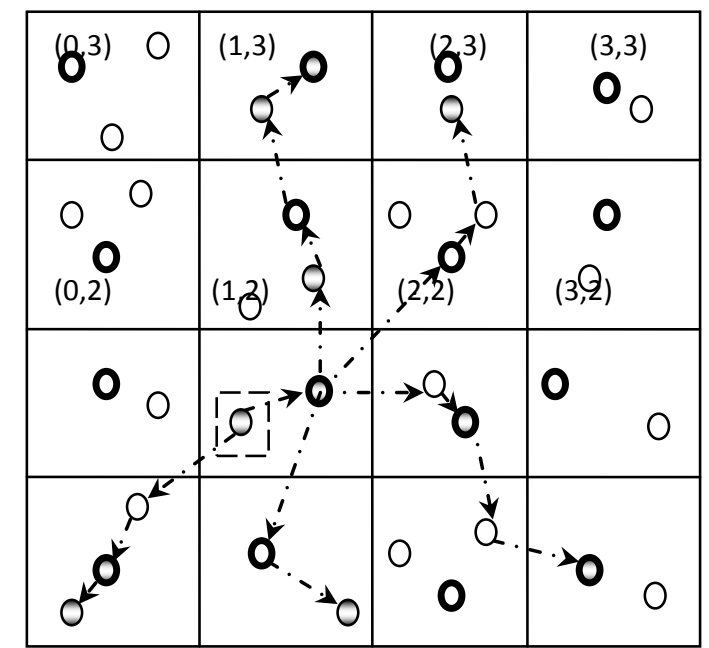

Multicast member

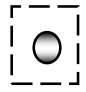

Multicast Source

O Zone representative

Fig 1: Highly Scalable zone based Multicast Protocol 
It incurs high overhead due to the large number of group members. For efficient packet forwarding, a multicast tree is constructed based on the set of multicast members to reach the destination node. This work undergoes bi-directional packet forwarding that forward the data in both upstream and downstream along the constructed tree in order to reach the destination. It totally reduces the communication overhead and access latency. In the upper layer, multicast data packets forward along the multicast tree in both upstream and downstream. In the lower layer, zone representative takes responsibility to receive packets from other zone representatives in the neighbor zone, and send them to the set of nodes in its local zone. In the proposed approach, location tracking service is incorporated with multicast zone membership management in order to reduce the overhead. This approach works under zone forwarding mode, where zone representative only can process the control messages or data packets related to membership management and packet forwarding.

In inter-zone multicast forwarding, a source node will forward the data packet to the zone representative of the target zone using id of the target node mentioned in the data packet. In order to reach the target, the source node finds the next hop to the target based on its neighbor table. This approach proposes next hop selection to determine the nearest neighbor node whose zone is the closest to the target zone than its own zone. In case, multiple next hop nodes are available to reach the target zone then determine the closest neighbor node to the target as the next hop. To determine the nearer neighbor node in the next hop, each node calculates the distance between its own zone $(\mathrm{x}, \mathrm{y})$ and the target zone $\left(\mathrm{X}_{\mathrm{tg}}, \mathrm{Ytgt}\right)$ as follows:

$$
\text { Distance }(\mathrm{X}, \mathrm{Y})=(\mathrm{X}-\mathrm{Xdst})^{2}+(\mathrm{Y}-\mathrm{Ydst})^{2}
$$

Thus, a zone with a smaller distance value is closer to the target zone is determined. Once determining the nearer next hop node to reach the target zone, the source node will forward the data packet to the zone representative of the target zone. On arrival at the destination zone, zone representative broadcast the data packet to the destination node in the zone by exploiting the information in the membership table.

\subsection{Group Membership Management}

The main aim of this work is to construct and maintain the network-wide zone-based bi-directional tree for multicast packet forwarding. In this approach, multicast tree is constructed using the location information tracked in reference to the zone rather than connecting the group member directly. It also deploys a location-aware quick group joining and leaving technique. In addition to, the proposed approach supports high scalability for a large group size. In the proposed approach, location information plays a main role in the multicast tree construction and group membership management mechanisms. Therefore, a node can quickly establish its connection to the tree when it is located along the transmission path of the multicast tree. In multicast tree construction, a source node in the multicast group $\mathrm{G}$ flood a beacon message Start Forward (G, Zone ID) in order to notify its existence to all of the nodes in the whole network. This message transmits the root zone ID of the zone where the source node is located.

\subsubsection{Joining procedure}

Each node in the network receives the beacon message from the source node, and they may have interest to join in the multicast group $\mathrm{G}$ for multicast forwarding. On receiving the beacon message, some of the nodes send a Join Req(G, Info, location) where $\mathrm{G}$ is the multicast group wants to join, Info is the message to its zone representative $\mathrm{M}$ and location is its own address. Then, zone representative initiates a representative joining procedure to process the request received from the nodes in the network. If Zonerep receives a request from the node in its own zone, adds the node to the list of downstream node in its multicast table. On the other hand, if the message is from any other zone then compare the zone depth of the requesting zone and its own zone. If the zone depth of the requesting member is smaller, add the requesting zone to its downstream zone list. Otherwise, zone representative forward the Join $\operatorname{Req}(\mathrm{G}$, Info, location) message towards the root zone.

\subsubsection{Leaving Procedure}

If a multicast member wants to leave a group $\mathrm{G}$, then it sends a Leave Req(Info, G) message to its zone representative. Zone representative leaves the member from its downstream node list after receiving a LEAVE message. Furthermore, Zonerep also removes the member from its multicast group when it does not receive the beacon message from the corresponding node within $2 *$ Intervalmax. In addition to, unused branches also removed from the multicast tree using Leave process.

\subsection{Adaptive Zone handling for the empty zone problem}

In the proposed approach, the constructed multicast tree is adjusted adaptively correspondingly to maintain consistency even under dynamic mobility. If the zone representative is moving out from its non-root zone, it is difficult to maintain the membership management. Therefore, Zonerep will send its multicast table to its upstream zone before leaving from its zone. Then, the upstream zone representative will take over all of its downstream zones and then remove the requesting zone from its downstream zone list using leave procedure. In order to notify the change in the upstream zone, then send Join Reply () messages to the entire newly added downstream zone. Then, newly added downstream zone changes its upstream zone ID after receiving the Join Reply () from its upstream zone. If the two upstream zones have the same distance from the root zone, one of the two upstream zones is randomly selected as an upstream zone. It leads to reliable route maintenance even under high dynamic mobility [14].

\subsection{Overhead Minimization in the proposed ZMRP Protocol}

This paper proposes two overhead reducing schemes: 1) Redundancy Reducing Flooding Mechanism (RR-FM), and 2) Beacon from Backup nodes Only (BBO). The RR-FM scheme introduces the efficient flooding mechanism in which redundant transmission of control messages is reduced. BBO scheme prompts the beacon only from the nodes located in the neighborhood of the multicast tree and revokes the remaining nodes from the beacon broadcast. Both schemes eliminate redundant messages in the network.

\subsubsection{Redundancy Reducing Flooding Mechanism (RR-FM)}

Control message from any node in the network is initially sent to its corresponding zone representative. The zone representative broadcast the message to all the identified nodes in its zone. The message header contains the list of identified members. Each receiving node of the control 
message identifies whether the list contains all its own neighbors. If any of its neighbors are missing from the list, receiving node attaches the missed neighbor ID in the list and forwards the message to the corresponding neighbor. Otherwise, the receiving node drops the message. This process continues till all the nodes in the network receive the control message [15]. This mechanism of the receiver list oriented zone based flooding avoids the redundant transmission of control messages, and it significantly reduces the resource consumption and collision in the network.

\subsubsection{Customizing Beacon Update Frequency}

Each node maintains a neighbor table through the BEACON message broadcast process. Periodic neighbor updating is unnecessary and it causes overhead in the network. Node should customize its beacon frequency based on the following criteria. If node mobility is greater than the threshold, it should update its position to neighbors frequently. If the node moves out of its current zone, it should update its location. All other nodes no need update their location frequently. This paper proposes a Beacon from Backup nodes Only (BBO) scheme, which involves the beacon from backup nodes only [16]. These backup nodes are the nodes located in the neighborhood area of the multicast tree. Backup nodes play a main role in forwarding the data when the routing path of the multicast tree fails. Nodes those are not located in the neighborhood of the multicast tree do not send the beacon periodically. Backup nodes overhear the data transmission by a multicast member over the multicast tree. Soon after overhearing the data packet transmission from the unknown multicast member, backup nodes broadcast a beacon. The unknown multicast member refers to the unidentified neighbor resides in the multicast tree. Now multicast nodes and backup nodes are aware of each other. Data forwarding failure of the existing multicast tree is repaired using such backup nodes. The location of the destination is piggybacked in the data packet, which assists the nodes to identify the location of the destination. It also assists to check whether the destination is located within their transmission range. If the destination is located within transmission range, expenditure of a beacon to identify the destination can be saved.

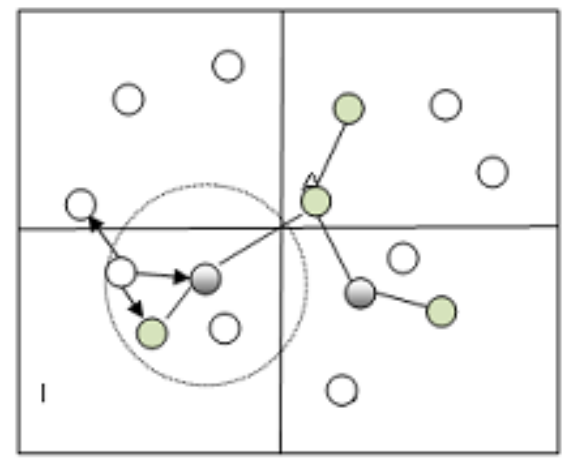

Multicast member

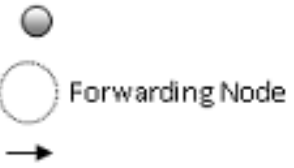

- Transmission range of Node $\mathrm{C}$

Fig 2: Backup node Beacon Broadcast
Figure 2 shows the beacon broadcast from the backup node. Data transmission of node $\mathrm{C}$ to node $\mathrm{E}$ is overheard by all the nodes $(\mathrm{H}$ and $\mathrm{F})$ in the transmission range of node $\mathrm{C}$. Link between Node $\mathrm{H}$ and node $\mathrm{C}$ is unidentified so far. So node $\mathrm{H}$ considers node $\mathrm{C}$ as a unknown node in the multicast tree. However, the link between Node $\mathrm{F}$ and $\mathrm{C}$ is identified earlier, and both are aware of each other. Hence, the node $\mathrm{H}$ alone broadcast a beacon message. Now link between Node $\mathrm{C}$ and $\mathrm{H}$ are unveiled, and both are aware of each other. Node I do not reside in the neighborhood of the multicast tree and do not overhear any data transmission. Therefore, Node I do not broadcast a beacon. In case of failure of the link between Node $\mathrm{C}$ and Node E, either Node H or F serves as backup nodes and assists seamless data delivery. BBO scheme reduces the unnecessary beacon from Node F and I.

\section{EXPERIMENTAL EVALUATION}

Quantitative analysis is carried out to measure the efficiency and scalability of the proposed HSZMP protocol using the simulation tool NS-2. Proposed protocol HSZMP is compared with well-known existing multicast protocol SPBM. Simulation is conducted with the following configuration parameters. To evaluate the scalability, network size is varied from $100 \mathrm{~m}$ X $100 \mathrm{~m}$ to $500 \mathrm{~m} \times 500 \mathrm{~m}$ with total nodes from 100 to 250 respectively while maintaining the same network density of 0.001 and with the varying transmission range from $25 \mathrm{~m}$ to $250 \mathrm{~m}$. Nodes speed varies from $1 \mathrm{~m} / \mathrm{s}$ to $20 \mathrm{~m} / \mathrm{s}$ with the random waypoint mobility model. IEEE 802.11 is chosen as a media access control protocol. User Datagram Protocol (UDP) is chosen as a transport agent and CBR is set as an application agent generates traffic at the rate varied from $10 \mathrm{Kbps}$. Each packet is 1280 bytes length. Overall simulation time is fixed as 500s.

\subsection{Experimental results}

The following section illustrates the experimental results of the proposed protocol in terms of packet delivery ratio, Average Delay, Data transmission Cost and Energy Consumption.

\subsubsection{Impact of Node Mobility on Packet Delivery ratio}

The Fig 3 illustrates the effect of mobility on the packet delivery ratio. To measure the efficiency of the protocol under a dynamic environment, the pause time is increased in the order of $3 \mathrm{~s}, 9 \mathrm{~s}, 21 \mathrm{~s}, 63 \mathrm{~s}$ and $189 \mathrm{~s}$. The packet delivery ratio of all the protocols decreases with the increasing mobility. Slope of the packet delivery ratio of the existing protocol SPBM is steeper when compared to the proposed HSZMP protocol. The overhead of membership maintenance in SPBM causes the collision in the network which leads to reduced packet delivery ratio under a high mobility conditions. Since, the proposed HSZMP protocol maintains the tree structure on the basis of the zone, even though the mobility is high it forwards the data over the multicast tree efficiently. Collision is avoided in the proposed protocol with the use of BPO scheme, and RR-FM scheme reduces the packet loss of the network, and also backup nodes assist in forwarding data during the link failure case. Hence, the proposed HSZMP protocol achieves high packet delivery ratio compared to SPBM.

The Fig 4 depicts the effect of network density on average delay for both SPBM and HSZMP. The average delay accounts the time consumption for the data delivery and also for the time taken for the member joining process to the multicast tree. 


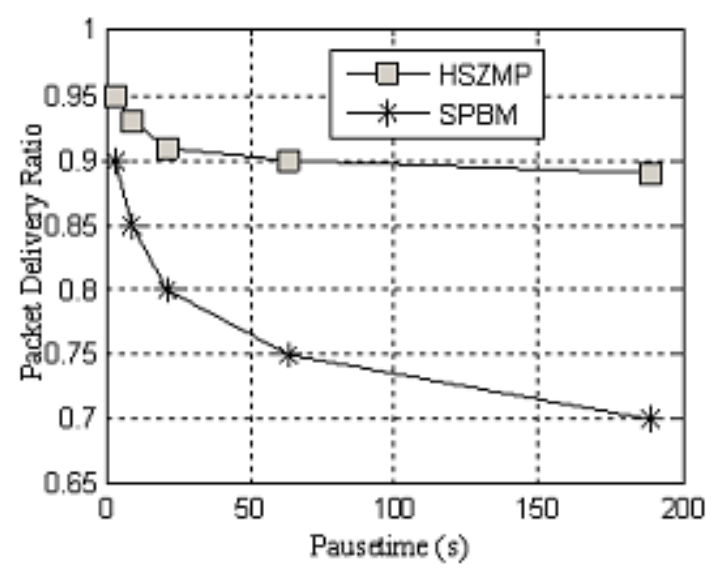

Fig 3: Packet Delivery Ratio of proposed HSZMP

\subsubsection{Impact of Network Density on Average Delay}

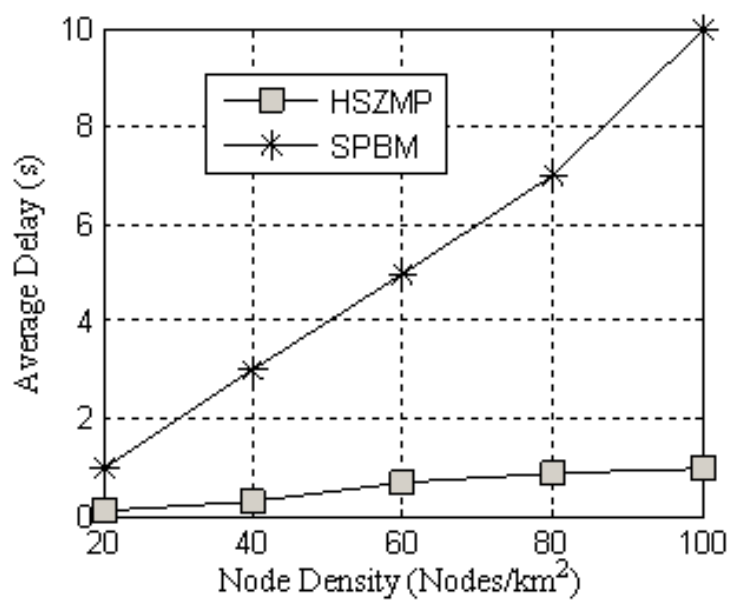

Fig 4: Average Delay of proposed HSZMP

Increment in the network density increases the average delay since the number of nodes involved in the data forwarding is higher in both protocols. In SPBM, the membership update process is done in multiple levels periodically. As quad-tree levels are maintained, membership change consumes high delay for the announcement of change in all levels. On the contrary, membership change is to the only zone representative. Since, membership management is done on a zone basis, the proposed HSZMP protocol achieves lesser delay when compared to all the existing protocol.

\subsubsection{Impact of Network size on Data transmission} Cost

Data transmission cost is the average number of control messages involved for each node, to transfer the data packet from multicast source to multicast destination. Fig 5 shows the comparative analysis of the data transmission cost of all the protocols for the various network sizes. All the protocols exhibit high cost when the network size increases. The proposed HSZMP protocol incurs less cost compared to SPBM.

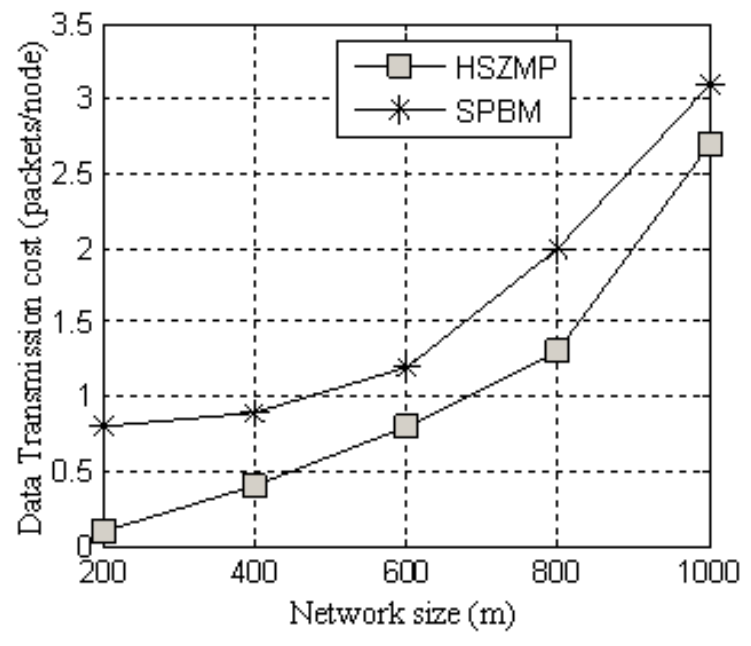

Fig 5: Data transmission Cost of proposed HSZMP

The reason is uses of customized beacon frequency, which reduces less beacon traffic in the network and also it invokes the RR-FM mechanism in which redundant control message is avoided. Tree maintenance is carried out on a zone basis also reduces the cost. In SPBM, Message flooding is done in multilevel over a quad tree, which significantly increases the cost. Therefore, the expense of data transmission cost is reduced in the proposed protocol, which improves the scalability.

\subsubsection{Impact of the number of nodes on Energy Consumption}

Energy Consumption reveals the amount of energy consumed by the entire network for the multicast during the entire simulation period. Fig 6 shows the comparison of energy consumption compared between proposed HSZMP protocol and existing protocol SPBM.

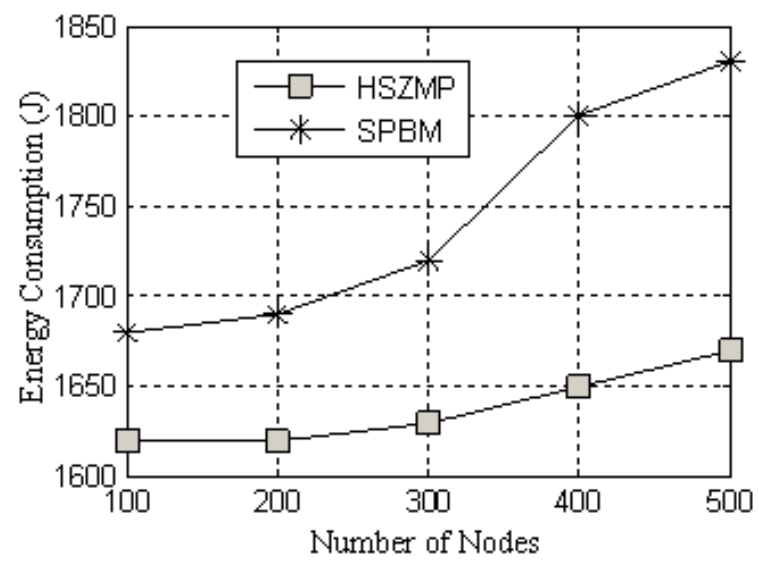

Fig 6: Energy Consumption

When the network range increases the number of nodes involved in the multicast tree increases which results in higher energy consumption. SPBM do not include the efficient flooding scheme of messages which increases energy consumption in the network. The proposed HSZMP protocol includes the efficient scheme for minimizing overhead such as RR-FM and BBO scheme. The energy expenditure on redundant transmission is avoided in the proposed system. As the energy required for periodic multilevel flooding used in 
SPBM is eliminated in the proposed HSZMP protocol, it achieves lesser energy consumption when compared to SPBM.

\section{CONCLUSION}

In this paper, a highly scalable zone based multicast protocol HSZMP for MANET is proposed. The proposed approach achieves high scalability by constructing a two-tier virtualzone-based structure using location aware mechanism. Therefore, the most important zone management and packet forwarding mechanism effectively perform their functions using the geometric information that reduced overhead for long path transmissions. The proposed HSZMP incorporates two schemes such as RR-FM and BBO to reduce the redundant overhead. The results obtained from the simulation reveals that the proposed protocol HSZMP achieves high packet delivery ratio, less average delay, less data transmission cost and reduced energy consumption when compared to SPBM.

\section{REFERENCES}

[1] C.C Chiang, "Wireless Network Multicasting", PhD thesis, University of California, Los Angeles, 1998.

[2] E. M. Royer and C. E. Perkins, "Multicast Operation of the Ad Hoc On-demand Distance Vector Routing Protocol", Proceedings of the ACM/IEEE International Conference on Mobile Computing and Networking, pp. 207-218, 1999.

[3] C. Wu, Y. Tay, "AMRIS: a multicast protocol for ad hoc wireless networks", Proceedings of the IEEE Military Communications Conference. pp 25-29, 1999.

[4] X. Zhang and L. Jacob, "Multicast zone routing protocol in mobile ad hoc wireless networks", Proceedings of the 28th Annual IEEE International Conference on Local Computer Networks, pp 150-159, 2003.

[5] C.-C. Chiang, M. Gerla, and L. Zhang, "Forwarding group multicast protocol (FGMP) for multi-hop mobile wireless networks", Cluster Computing, Vol. 1, No. 2, pp. 187196,1998 .

[6] J. J. Garcia-Luna-Aceves and E. Madruga, "The Coreassisted Mesh Protocol", IEEE Journal on Selected Areas in Communications, Vol 17 , No 8, pp 1380-1394, 1999.
[7] S. Bae, S. Lee, W. Su and M. Gerla, "The Design, Implementation, and Performance Evaluation of the On demand Multicast Routing Protocol in Multi-hop Wireless Networks," IEEE Network, Vol. 4, No. 1, pp. 70-77, 2002.

[8] M. Mauve, H. Fubler, J. Widmer, and T. Lang, "Positionbased multicast routing for mobile ad-hoc networks", ACM SIGMOBILE Mobile Computing and Communications Review, Vol 7, No 3, pp 53-55, 2003.

[9] Pariza Kamboj, A.K.Sharma "Scalable Energy Efficient Location Aware Multicast Protocol for MANET (SEELAMP)" Journal of Computing, Vol 2, No 5, 2010.

[10] Y. B. Ko and N. Vaidya, "Geocasting in Mobile ad hoc networks: location based multicast algorithms", Proceedings of the Second IEEE Workshop on Mobile Computing Systems and Applications, pp 101-110, 1999.

[11]Young-Bae Ko, Nitin H. Vaidya, "GeoTORA: A Protocol for Geocasting in Mobile Ad Hoc Networks", Proceedings of the International Conference on Network Protocols, pp 240-250, 2000.

[12] S S.M.Das, H. Pucha and Y.C. Hu, "Distributed Hashing for Scalable Multicast in Wireless Ad Hoc Network" In IEEE Transactions on Parallel and Distributed Systems Vol 19, No 3, pp 347-362, 2008.

[13] M. Transier, H. Fubler, J. Widmer, M. Mauve, and W. Effelsberg, "A Hierarchical Approach to Position-Based Multicast for Mobile Ad-hoc Network", Wireless Networks, Vol. 13, No 4, pp. 447-460, 2007.

[14] X. Xiang and X. Wang "An Efficient Geographic Multicast Protocol for Mobile Ad Hoc Networks" In IEEE International Symposium on a World of Wireless, Mobile and Multimedia Networks (WoWMoM), Niagara-Falls, Buffalo, New York, June 2006

[15] H. Lim and C. Kim, "Multicast Tree Construction and Flooding in Wireless Ad Hoc Networks," In Proc. of the ACM Int'l Workshop on Modeling, Analysis and Simulation of Wireless and Mobile System (MSWIM), pp 61-68, Aug. 2000.

[16] Quanjun Chen, Salil S. Kanhere, and Mahbub Hassan, "Adaptive Position Update for Geographic Routing in Mobile Ad-hoc Networks" IEEE Transactions on Mobile Computing, Vol 12, No 3, pp 489-501, 2013. 